\title{
SCHEMATIZATION OF INFINITE SETS OF REWRITE RULES. APPLICATION TO THE DIVERGENCE OF COMPLETION PROCESSES
}

\author{
Hélène Kirchner \\ Centre de Recherche en Informatique de Nancy \\ BP 239 Vandoeuvre-les-Nancy Cedex
}

FRANCE

\section{Introduction}

This study was originally motivated by the divergence problem of the completion procedure for term revititing system $[17,18,11]$. The practical interest of a completion procedure is limited by the fact that it can generate infinite sets of rewrite rules. Moreover the uniqueness of the result of the completion procedure, given a fixed ordering for orienting equations, implies that it cannot be expected to find another completion strategy for which the completion terminates.

There are two kinds of divergence:

- The first one (in depth) is due for instance to the existence of a rule 1->I that can be superposed successively on a rule $1,->r_{1}$, then on the rule $1_{2}->r_{2}$ deduced from this superposition and so on. Such cases have been studied by Hermann and Privara who give in [7] sufficient criterion for detecting a priori this kind of divergence.

- The second kind of divergence (in breadth) occurs only in equational completion procedures and is due to the existence of infinite complete sets of unifiers modulo a set of equations.

A first approach to deal with the divergence problem has been proposed in [3]. Rules generated by the completion process are dynamically splitted into different sequences according to some regularity criteria, such as a recurrence relation or a same origin. Then lemmas are asked to the user in order to make these pairs confluent. The problem of finding recurrence relation between terms in a sequence has been studied in [12] and [14].

The goal of that paper is neither to discover recurrence relations in a set of rules generated by completion, nor to predict a priori the divergence of completion, but rather to propose a formalism to deal with the problem of divergence, namely the definition of meta-rules. This paper is an attempt to give answers for different questions:

1) given an infinite set of rules, the first problem is to find a finite set of schemas, here called meta-rules, where some variables, called meta-variables, may have infinite sets of possible values. Discovering the schemas and the domains of meta-variables is yet a matter of heuristics and we propose a method here.

2) The main problem is to be able to use meta-rules for deciding the validity or satisfiability of an equation in the equational theory defined by the infinite set of rules. We determine here precise conditions that have to be checked in order to get a sound and complete schematization of the infinite set of rules. Soundness and completeness ensure that it is equivalent to use the set of meta-rules and the infinite 
set of rules.

3) Of course a preliminary definition of how to use meta-rules must be given. A meta-rewriting relation is introduced and appears to be very similar to other appraaches introducing constrained variables in rewriting. We also explore in which cases meta-rewriting coincides with equational rewriting and when a Church-Rosser property for meta-rewriting can be decided.

4) Meta-rules can be used to solve equations, with a narrowing-like process. A meta-narrowing relation is defined and studied. It allows dealing with infinite narrowing trees and infinite complete sets of solutions. Each meta-solution describes an infinite sequence of solutions.

The next section gives examples of divergence problems in order to support intuition for the definition of meta-rules given in section 3. Meta-rewriting is studied in section 4 and meta-narrowing in section 5 .

\section{Some examples}

Let us consider some simple but significant examples to illustrate which kind of infinite rewrite rule system we want to handle.

Example 1 : Signed binary trees: let us consider the following set of rewrite rules:

$-(-(x)) \rightarrow x$

$-(f(x, y)) \rightarrow f(-(y),-(x))$

$f(f(y, x),-x) \rightarrow y$

$f(-x, f(x, y)) \rightarrow y$

Starting from these rules, the completion procedure generates two infinite families of rules:

$$
\begin{array}{ll}
\{f(-x, f(x, y))->y, & f\left(f\left(-x_{2},-x_{1}\right), f\left(f\left(x_{1}, x_{2}\right), y\right)\right)->y, \\
f\left(f\left(-x_{2}, x_{1}\right), f\left(f\left(-x_{1}, x_{2}\right), y\right)\right)->y, & \left.f\left(f\left(x_{2},-x_{1}\right), f\left(f\left(x_{1},-x_{2}\right), y\right)\right)->y \ldots\right\} \\
\{f(f(y, x),-x) \rightarrow y, & f\left(f\left(y, f\left(x_{1}, x_{2}\right)\right), f\left(-x_{2},-x_{1}\right)\right)->y, \\
f\left(f\left(y, f\left(-x_{1}, x_{2}\right)\right), f\left(-x_{2}, x_{1}\right)\right)->y, & \left.f\left(f\left(y, f\left(x_{1},-x_{2}\right)\right), f\left(x_{2},-x_{1}\right)\right)->y \ldots\right\}
\end{array}
$$

Example 2: Associativity and idempotency: the equations

$$
\begin{aligned}
& f(f(x, y), z)=f(x, f(y, z)) \\
& f(x, x)=x
\end{aligned}
$$

directed from left to right, generate two families of rules:

$$
\begin{aligned}
& \left\{f \left(x_{1}, f\left(x_{2}, \ldots, f\left(x_{k}, f\left(x_{1}, \ldots, f\left(x_{k}, z\right) \ldots\right) \rightarrow f\left(x_{1}, f\left(x_{2}, \ldots, f\left(x_{k}, z\right) \ldots\right)\right\}\right.\right.\right.\right. \\
& \text { and } f\left(x_{1}, f\left(x_{2}, \ldots, f\left(x_{k}, f\left(x_{1}, \ldots, f\left(x_{k-1}, x_{k}\right) \ldots\right) \rightarrow f\left(x_{1}, f\left(x_{2}, \ldots, f\left(x_{k-1}, x_{k}\right) \ldots\right)\right\}\right.\right.\right.
\end{aligned}
$$

Example 3 : One rule is enough to generate an infinite family $[1,4]$. The rule $f(g(f(x)))->g(f(x))$ generates the infinite family

$$
\left\{f\left(g\left(f\left(x_{1}\right)\right)\right) \rightarrow g\left(f\left(x_{1}\right)\right)\right\}
$$

Example 4: Another type of divergence can be caused by infinite sets of unifiers.

$$
\begin{aligned}
E: & -(-(x))=x \\
& -(f(x, y))=f(-(y),-(x)) \\
& -h(x)=h(-x)
\end{aligned}
$$




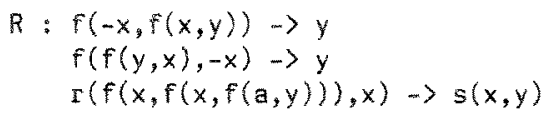

Superposition modula $E$ of the rules $r(f(x, f(x, f(a, y))), x) \rightarrow s(x, y)$ and $f(-x, f(x, y)) \rightarrow y$, generates the infinite family of rules:

$r\left(f(a, y), f\left(x_{1},-x_{1}\right)\right)->s\left(f\left(x_{1},-x_{1}\right), y\right), \quad r\left(f(a, y), h\left(f\left(x_{1},-x_{1}\right)\right)\right)->s\left(h\left(f\left(x_{1},-x_{1}\right)\right), y\right)$, $\left.\Gamma\left(f(a, y), h\left(h\left(f\left(x_{1},-x_{1}\right)\right)\right)\right)->s\left(h\left(h\left(f\left(x_{1},-x_{1}\right)\right)\right), y\right) \ldots\right)$

due to the fact that unifying modulo $E$ the two terms $f(x, f(x, f(a, y)))$ and $f\left(-x^{\prime}, f\left(x^{\prime}, y^{\prime}\right)\right)$ results in an infinite complete set of unifiers. The values for the variable $x$ in these substitutions are solutions of the equation $x=-x$ :

$$
\left\{\left(x^{\leftarrow}-f\left(x_{1},-x_{1}\right)\right),\left(x^{\leftarrow} h\left(f\left(x_{1},-x_{1}\right)\right)\right),\left(x^{\leftarrow} h\left(h\left(f\left(x_{1},-x_{1}\right)\right)\right), \ldots\right\}\right.
$$

We now examine some characteristics of these examples.

- First there exists a generalization $G$ of the sequence of left-hand sides $l_{k}$ of the rules modulo an equivalence relation denoted $\sim$. That means that for any $I_{k}^{k}$, there exists a substitution $\sigma_{k}$ such that $\sigma_{k}(G) \sim 1_{k}$.

- Second, it is possibie to define, from the substitutions $\sigma_{k}$, a set of terms that can be described either by a structural property, or as at of solutions of an equation.

Example 5 : (Example 2 con't: associativity and idempotency)

Let us consider the equivalence $\sim$ generated by the rewrite system:

$R_{0}: f(f(x, y), z) \rightarrow f(x, f(y, z))$

The sequence of left-hand sides $1_{k}$ of the first family of generated rules can be generalized modulo $\sim$ by the term $G=f(x, f(x, z))$, where $x$ can be substituted by any value in the set of terms $\left\{x_{1}, f\left(x_{1}, x_{2}\right), f\left(x_{1}, f\left(x_{2}, x_{3}\right)\right), f\left(x_{1}, f\left(x_{2}, f\left(x_{3}, x_{4}\right)\right)\right) \ldots\right\}$.

Example 6 : (Example 3 con't: only one rule)

$\sim$ is here the syntactic equality and the left-hand sides are generalized by the term $f(g(x))$. The set of substituted values for $x$ is

$\left\{f\left(x_{1}\right), g\left(f\left(x_{1}\right)\right), g\left(g\left(f\left(x_{1}\right)\right)\right), \ldots\right\}$.

Example I : (Example 4 con't: infinite set of unifiers) The sequence of left-hand sides $I_{k}$ can be generalized, modulo the equivalence generated by the set of equations $E$, ${ }^{b y}$ the term $G=r\left(f\left(a_{k} y\right), x\right)$, since for any $l_{k}$, there exists a substitution $o_{k}$ s.t. $\sigma_{k}(g) \sim 1_{k}$, with $\sigma_{k}=h^{k}\left(f\left(x_{1},-x_{1}\right)\right)$.

\section{Definition of meta-rules}

Notations are consistent with those in [8] for instance. Classically $T(F, V)$ denotes the free $F$-algebra generated by the set of variables $V$, whose elements are called terms. We also need the notion of recursive set of terms: that means that there exists an algorithm allowing to decide whether a term belongs to that set. Of course a finite intersection of recursive sets is a recursive set.

Definition 1: Let DOM $=\left\{\mathrm{DOM}_{\dot{1}} \mid \dot{i}\right.$ in $\left.\mathrm{I}\right\}$ an indexed family of recursive subsets of $T(F, V)$. A set of meta-variables $M V=\{M V, \mid i$ in $I\}$ is an indexed family of variable sets associated with DOM. A function $d: d(X)=D O M_{i}$ maps each meta-variable $X$ in $M V_{i}$ to its domain $\mathrm{DOM}_{\mathrm{i}}$. 
Note that we could consider variables as meta-variables whose associated domain is $T(F, V)$. However it is clearer to distinguish them and we denote in what follows variables by lower case letters $x, y, z \ldots$ and meta-variables by upper case letters $X, Y, Z$.

On each DOM there is an equivalence relation $\sim_{j}$. For any terms $t$ and $t$, in $T(F, V)$, we define ' the relation $t|-| t$ ' iff there exists $i$ in I such that $t, t$ ' are in DOM. and $t \sim_{i} t$ '. Then $\sim$ is the smallest congruence closed by substitutions generated by $1-1$.

In the cases we consider here, it is possible to find a generating set for each DOM in the following sense:

Definition 2 : $D^{\circ} M_{\text {i }}$ is generated by a set of structures $S_{i}$ included into $D O M$. iff $\overline{D O M}$ is the set of terms $t$ such that there exist a term $\frac{i}{s}$ in $s_{i}$ and a substitution $\sigma$ of $\dot{t}(F, V)$ satisfying $t \sim_{i} \sigma(s)$.

Definition 3: A meta-tere is an element of the free F-algebra generated by $V$ and $M V$ and denoted $T(F, V U M V)$.

Meta-terms are denoted by upper case letters $T, U, G, D \ldots$ If $T$ is a meta-term, $M V(T)$ denotes the set of its variables and meta-variables. Terms can be substituted for meta-variables under some condition.

Definition 4 : An instantiation of meta-variables $\phi$ is a family of mappings

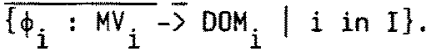

Whenever DOM ${ }_{i}$ is generated by a set of structures $S_{i}$, it is possible to define an additional notion of principal instantiation:

Definition 5 : Let $S=\left\{S_{i} \mid i\right.$ in $\left.I\right\}$ such that $D O M_{i}$ is generated by $S_{i}$. A principal instantiation of meta-variables $\psi$ is a family of mappings $\left\{\psi_{i}: M V_{i}-j s_{i} \mid\right.$ in $\left.I\right\}$.

By hypothesis on the structures, any instantiation of meta-variables can be decomposed into a substitution of $T(F, V)$ and a principal instantiation, modulo the equivalence $\sim$. This decomposition extends to any instantiation of variables and meta-variables. More precisely:

Lemma 1 : For any meta-term $T$ and any instantiation $\phi$ of $M V(T)$, there exists a substitution $Y$ of $T(F, V)$ and a principal instantiation $\psi$ of meta-variables of $T$ such that $\phi \sim \gamma \cdot \psi[M V(T)]$ (where . is composition of functions).

Example 8: (Example 3 con't: only one rule)

Let us consider the meta-term $T=g(g(X, Y), x)$ and the instantiation $\phi=\left(X \leftarrow f\left(t_{1}\right)\left(Y \leftarrow f\left(t_{2}\right)\right)\left(x \leftarrow t_{3}\right)\right.$. We have then

$\psi=\left(X \leftarrow f\left(x_{1}\right)\right)\left(Y \leftarrow f\left(x_{2}\right)\right)$ and $y=\left(x_{1} \leftarrow t_{1}\right)\left(x_{2} \leftarrow t_{2}\right)\left(x \leftarrow t_{3}\right)$.

The definition and an empiric construction of meta-rules are now given.

Definition 6 : A meta-rule is a directed pair of meta-terms denoted G->D such that MV $(G)$ contains MV $(D)$. A meta-rule $G->D$ is said to be unconstrained iff for any meta-variable $X$ in $M V(G), d(X)=T(F, V)$. A set of meta-rules is said unconstrained if $f$ all the meta-rules are unconstrained.

A question obviously arises now: how to find a meta-rule? Unfortunately we are only able at this point to propose some heuristics. 
- The first thing to do is to find together an equivalence relation $\sim$ and the skeleton of a sequence of rules by generalization modulo $\sim$ of all the elements of the sequence.

- Each variable introduced by generalization is a meta-variable. The set of structures associated with that meta-variable is given by the different instances of the variable in the sequence. The domain is determined by the set of terms which the variables may be instantiated to, in order to apply a rule of the sequence.

Let us see on our examples what are the meta-rules.

Example 9 : (Example 1 con't) In the signed binary trees theory, the congruence $\sim$ is generated by the set of rules

$$
\begin{aligned}
R_{0}: & -(-(x)) \rightarrow x \\
& -(f(x, y)) \rightarrow f(-(y),-(x))
\end{aligned}
$$

There are two unconstrained meta-rules:

$$
f(-x, f(x, y)) \rightarrow y \text { and } f(f(y, x),-x) \rightarrow y \text {. }
$$

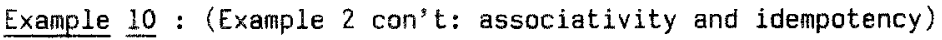

To each family is associated an unconstrained meta-rule:

$$
f(x, f(x, z)) \rightarrow f(x, z) \text { and } f(x, x) \rightarrow x .
$$

Example 11: (Example 3 con't) The family of rules $\left\{f\left(g^{i}\left(f\left(x_{1}\right)\right)\right)->g^{i}\left(f\left(x_{1}\right)\right)\right\}$ is represented by the meta-rule $f(g(X)) \rightarrow g(X)$.

The domain of $x$ is here the set of terms with top symbol $f$ or $g$.

Example 12: (Example 4 con't: infinite set of unifiers)

The meta-rule is $r(f(a, y), X) \rightarrow s(x, y)$. The domain of $x$ is the set of all the solutions modula $E$ of the equation $(x=-x)$.

But to be sound, the schematization must satisfy some conditions.

Definition I : An infinite rewrite rule system Roo is schematized by a set of wetarules MR iff

(a) $\sim$ is included into the congruence $<-*->R_{\infty}$ generated by $R_{\infty}$.

(b) for any meta-rule G->D of MR, any principal instantiation $\psi$ of its metavariables, $\psi(G)<-*->R_{\infty} \psi(D)$.

Example 13: An example where this definition is not satisfied is the following one. The rules $f(x+e) \rightarrow f(x)$ and $(x+y)+z \rightarrow x+(y+z)$ generate the set of rules

$$
\{f(x+e) \rightarrow f(x), f(x+(y+e)) \rightarrow f(x+y), f(x+(y+(z+e)) \rightarrow f(x+(y+z)) \ldots\} \text {. }
$$

In the sequence of left-hand sides, the subterms at occurrence 1 give a sequence $\{x+e, x+(y+z), x+(y+(z+e)) \ldots\}$ that can be generalized by the meta-term $x+e$ with DOM generated by $S=\{x, x+y, x+(y+z) \ldots\}$. Nevertheless the introduction of $(x+e) \rightarrow x$ would completely modify the equational theory, which is not the case with the metam rule $f(x+e) \rightarrow f(x)$.

Some sufficient conditions to ensure the condition (b) in the previous definition can be given in the case of only one set of meta-variables:

- if the set of structures contains a variable, (b) is equivalent to:

$\left(b_{1}\right)$ for any meta-rule $G->0, G<-* \rightarrow R_{\infty} D$.

$-{ }_{\text {if }}$ for any principal instantiation $\psi$, there exists $j$ such that $\psi=\psi_{0}^{j}$, where $\psi_{0}$ is a principal instantiation, (b) is equivalent to:

$\left(b_{2}\right)$ for any meta-rule $G->0, \psi_{0}(G)\left\langle-*->R_{\infty} \psi_{0}(D)\right.$.

That results from the stability of the congruence $\langle-*-\rangle R_{\infty}$ by substitutions. 
- more generally, if for any principal instantiation $\psi$, there exist an integer $j$ and a context $c$ such that $\psi=C^{J}\left(\psi_{0}\right)$, where $\psi_{0}$ is a principal instantiation, (b) is equivalent to:

$\left(b_{3}\right)$ for any meta-rule $G->D, \psi_{0}(G)<-*->$ Rio $\psi_{0}(D)$.

That results from the stability of the congruence $<-*->R_{0}$ by context.

Notice that anyway this condition (b) only involves principal instantiations but not any instantiation.

The reader is invited to check that in all our examples, the conditions of the definition of schematization are fulfilled.

\section{Meta-rewriting}

Once a schematization has been found, we must be able to use it, that is to rewrite with meta-rules. In general, the usual notion of rewriting modulo $\sim$ does not work. To illustrate this problem, let us consider the following example.

Example 14 : (Example 3 con't) In the meta-rule $f(g(x)) \rightarrow g(x)$ of the one rule example, the domain of $X$ is the set of terms with top symbol $f$ or $g$. Notice that if "a" is a constant, the terms $f(g(a))$ and $g(a)$ satisfy $f(g(a)) \rightarrow g(a)$ if the meta-rule is used as an ordinary rewrite rule. However $f(g(a))$ and $g(a)$ are not equivalent in the theory and thus are never Roo-equivalent. Actually the constraint on the meta-variable $X$ has been forgotten when matching $f(g(x))$ to $f(g(a))$. Indeed the value "a" is not allowed for $X$ since it does not belong to the domain of $X$.

This leads to define another rewriting relation, called meta-rewriting, which takes into account the domains of the meta-variables. Before defining how to reduce meta-terms with meta-rules, we first have to introduce convenient notions of equality and matching on meta-terms. The meta-equality is the congruence defined on $T(F, V M M)$ as follows:

Definition 8 : Two meta-terms $T_{1}$ and $T_{2}$ are meta-equal (denoted $T_{1} \equiv T_{2}$ ) iff for any instantiation $\phi$ of their meta-variables, $\phi\left(T_{1}\right) \sim \phi\left(T_{2}\right)$.

It is clear that the restriction of the meta-equality to terms without metavariables is the congruence - In all our examples, it is possible to decide metaequality. First it is possible to restrict the definition of meta-equality to principal instantiations:

Lemma 2 : Two meta-terms $T_{1}$ and $T_{2}$ are meta-equal (denoted $T_{1} \equiv T_{2}$ ) iff for any principal instantiation $\psi$ of their meta-variables, $\psi\left(T_{1}\right) \sim \psi\left(T_{2}\right)$.

Example 15: (Example 1 and 2 con't) In the signed binary trees example and in the associativity and idempotency example, since $D O M=\{T(F, V)\}, T_{1} \equiv T_{2}$ iff $T_{1} \sim T_{2}$.

Example 16: (Example 3 con't $^{\prime}$ ) In the one rule example, an easy structural induction proves that meta-equality is syntactic equality: $T_{1} \equiv T_{2}$ iff $T_{1}=T_{2}$.

Example 17: (Example 4 con't) In the example with an infinite set of unifiers, the decision procedure is more complex and is composed of three successive steps:

- compute the E-normal form of $T_{1}$ and $T_{2}$

- apply the rule $-x \rightarrow X$ on the meta-variables

- check the syntactic equality of the two resulting terms, 
Meta-matching is a generalization of matching taking into account the metaequality and the domain of meta-variables.

Definition 9 : Let $T$ and $T$ ' two meta-terms in $T(F, V M V)$. A weta-match from $T$ to $T$ ' is a substitution $\sigma^{\sim}$ on $T(F, V U M V)$ such that

$-\sigma^{2}(T) \equiv T^{\prime}$

- for any meta-variable $x$ in the domain of $\sigma^{\sim}$ such that $d(X)=D O M_{i}$, for any instantiation $\phi, \phi\left(\sigma^{*}(X)\right)$ belongs to DOM $i$.

Example 18: (Example 3 con't) In the one rule example, there is no meta-match from $\overline{f(g(x))}$ to $f(g(a))$, because of the definition of $d(x)$. But $(x \leftarrow f(a))$ is a meta-match from $f(g(X))$ to $f\left(g(f(a))\right.$ and $\left(X^{\leftarrow}-f\left(X^{\prime}\right)\right)$ is a meta-match from $f(g(X))$ to $f\left(g\left(f\left(X^{\prime}\right)\right)\right.$.

Note that whenever $D O M=\{T(F, V)\}$, any match modulo $\sim$ is a meta-match. So there may exist in general a meta-match but no match, and there may exist in general several meta-matches. Examples 1 and 2 (signed binary trees, associativity and idempotency) illustrate this simplest situation. In the one rule example, meta-matching consists of syntactic matching plus a check on the substituted values for metavariables. For the example of an infinite set of unifiers, one can design a matching algorithm based on E-matching, the meta-equality decision procedure and the check that any value substituted for a meta-variable is a solution of the equation $(x=-x)$.

We are now able to define meta-rewriting.

Definition 10: The weta-rewriting relation with a set $M R$ of meta-rules, denoted $==>$, is defined on $T(F, V M V)$ by: $T==>\left[U, \sigma^{-}, G->D\right] T$ '

iff - $G->D$ belongs to MR,

- $\sigma^{\sim}$ is a meta-match from $G$ to $T / u$

$\left(T\right.$, is obtained by replacing the subterm of $T$ at occurrence $u$ by $\left.\sigma^{\sim}(D)\right)$.

Let $=\Rightarrow \gg$ be the relation defined on meta-terms by $T=z \gg T$, iff there exists $T$ such that $T \Rightarrow T_{1} \equiv T^{\prime}$.

Let $\langle\langle=\# \Rightarrow \gg$ be the reflexive, symmetric and transitive closure of $=\Rightarrow\rangle$.

Assume that $M R$ is a schematization of an infinite set of rules Roo. We get then by Lemma 1 and Definition 8 :

Proposition 1 : Let $T$ and $T^{\prime}$ be two meta-terms and MR a schematization of Ros. Then $T\langle\langle=* \Rightarrow\rangle\rangle$ ' implies that for any principal instantiation $\psi$ of $T$ and $T$, , $\psi(T)<-*->R_{\infty} \psi\left(T^{\prime}\right)$.

An easy corollary of this proposition is that for two terms without metavariables $t$ and $t$ ', if $t\langle\langle=* \Rightarrow\rangle\rangle t^{\prime}$ then $t\langle-*-\rangle R_{\infty} t^{\prime}$. This property ensures the soundness of the schematization. But we usually want more, since we want that the set of meta-rules allows deciding the equality in the theory. That leads to the notion of completeness of the schematization.

Definition 11 : The schematization of Roo with MR is complete iff $<-*->$ Ro coincides with the restriction of $\langle\langle=* \Rightarrow\rangle$ to terms without meta-variables, denoted $\langle\langle-*-\rangle\rangle$.

With regard to the problem of divergence of the completion procedure, we already know $[9,11]$ that, given an original set of equations $A$, the congruence generated by $A$ coincides with $\left\langle-*->R_{m}\right.$. We can thus characterize a complete schematization: 
Proposition 2 : The schematization of $R_{\infty}$ is complete iff for any equation $g=d$ of $A$,

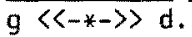

The reader is invited to check that the given examples satisfy this condition. One can find in [16] another characterization of complete schematization, independent from the notion of instantiation, and based on the Church-Rosser property of $=\Rightarrow \gg$, defined below.

In order to effectively use meta-rewriting for checking Rom-equality on terms, it is important to have a Church-Rosser property for $=\Rightarrow\rangle$. Then $\langle\langle-*-\rangle\rangle$ will be easily decidable if $=\Rightarrow>$ satisfies the following definition.

Definition 12 : A set of meta-rules MR is convergent iff the relation $=\Rightarrow \gg$ is noetherian and satisfies the Church-Rosser property:

for any meta-terms $T$ and $T^{\prime}$ such that $T\left\langle\langle=* \Rightarrow\rangle T^{\prime}\right.$, there exists a meta-term $T^{\prime \prime}$ such that $T=* \Rightarrow \gg T^{\prime \prime}$ and $T{ }^{\prime}=* \Rightarrow>T^{\prime \prime}$.

For an unconstrained set of meta-rules, remind that the relation $=\Rightarrow>$ coincides with equational rewriting modulo $\sim$. In that case, an equational completion procedure allows proving the Church-Rosser property.

Example 19: (Example 1 con't) In the signed binary trees theory, the meta-rules are unconstrained and the equational completion procedure modulo $R_{0}$ allows proving the Church-Rosser property of that system, although equivalence classes modulo $R_{0}$ are not finite [14].

Example 20: (Example 2 con't: associativity and idempotency)

The infinite set of generated rules has been schematized by a set of two unconstrained meta-rules:

$f(x, f(x, z)) \rightarrow f(x, z)$ and $f(x, x) \rightarrow x$.

We can thus use an equational completion procedure modulo the associativity axiom for $f$. The first rule can be reduced modulo associativity by the second one and thus the set of meta-rules allowing to decide equality in this theory is reduced to the unique meta-rule $f(X, X) \rightarrow X$. Note that in this particular case, we have been able to complete the system without using associative unification for which infinite complete sets of unifiers can appear.

Example 21: (Example 3 con't) The system of meta-rules reduced to the only meta-rule $f(g(X)) \rightarrow g(X)$ can be proved convergent.

Example 22 : (Example 4 con't: infinite set of unifiers) A completion process can be designed that generates first the meta-rule $r(f(a, y), X)) \rightarrow s(x, y)$ as already seen, then by superposition of the first rule in this meta-rule at occurrence 1 , another meta-rule $r(z, X) \rightarrow s(X, f(-a, z))$. The previous meta-rule then disappears and we get so a convergent system of meta-rules.

\section{Meta-narrowing}

In this section we define the meta-narrowing relation and a meta-narrowing process that allows solving equations when the usual narrowing process $[6,10,19]$ diverges. Actually we will be able to handle two kinds of problems:

- Assume that we want to solve equations in an equational theory for which an infinite convergent term rewriting system is known. The usual process of narrowing is 
sound and complete, but in practice very inefficient. Moreover special strategies must be adopted in that case to avoid infinite narrowing derivations. But whenever it is possible to find a set of meta-rules that is a complete schematization for this infinite term rewriting system, a complete set of solutions for an equation in this theory can be found, as shown hereafter.

- The second kind of problems is again the divergence of the narrowing procedure. As for the completion procedure, the process can diverge either in depth or in breadth for equational narrowing. Then the process generates an infinite set of goal rules [19] that can be splitted into sequences for which a meta-rule can be found. The same notion of schematization may be applied, but it must be noticed that now families of goal rules are schematized.

The two previous kinds of problems are actually handled in a uniform framework of meta-terms, which allows both a set of meta-rules for defining the equational theory and goal rules described by meta-terms. A meta-narrowing process applied to metaterms allows finding a set of meta-solutions, from which a complete set of solutions of the initial equation to solve is deduced, by instantiation of meta-variables. So assume from now on that the equational theory described by the set of axioms Roo is schematized by a set of meta-rules MR.

The definition of a meta-narrowing relation on meta-terms first needs to define the notion of meta-unification.

Definition $13:$ let $T$ and $T$ ' two meta-terms in $T(F$, WMV). A meta-unifier of $T$ and $T$ ' is a substitution $\sigma^{\sim}$ on $T(F, V M V)$ such that

$-\sigma^{\sim}(T) \equiv \sigma^{\sim}\left(T^{\prime}\right)$

- for any meta-variable $x$ in the domain of $\sigma^{\sim}$ such that $d(X)=D O M_{i}$, for any instantiation $\phi, \phi\left(\sigma^{\sim}(X)\right)$ belongs to $\mathrm{DOM}_{i}$.

Whenever DOM $=\{T(f, V)\}$, a meta-unifier is an unifier modulo $\sim$. There are in general several unifiers and we can define as usual a generating subset of the set of meta-unifiers.

Definition 14: A complete set of meta-unifiers of two meta-terms $T$ and $T$ ' is a set denoted Meta- Unif $\left(T, T^{\prime}\right)$ of substitutions of $T(F, V U M V)$ satisfying:

(1) for any $\sigma^{\sim}$ in Meta-Unif $\left(T, T^{*}\right)$, for any $X$ in the domain of $\sigma^{\sim}$, the variables and meta-variables of $\sigma^{\sim}(X)$ can be chosen distinct from those in MV $(T) U M V\left(T^{\prime}\right)$.

(2) any substitution in Meta-Unif( $T, T$ ') is a meta-unifier of $T$ and $T$ '.

(3) for any meta-unifier $a^{-}$of $T$ and $T^{\prime}$, there exists $\sigma^{\sim}$ in Meta-Unif $\left(T, T^{\prime}\right)$ such that $\sigma^{\sim} \leqq a^{\sim}$ [MV(T)UMV(T')] (which means that there exists $\beta^{\sim}$ such that for any $X$ in $\left.\operatorname{MV}(T) \operatorname{UMV}^{\prime}\left(T^{\prime}\right), \beta^{-}\left(\sigma^{\prime \prime}(X)\right) \equiv a^{\alpha}(X)\right)$.

Again there is not in general a unique element in a complete set of metaunifiers. Beyond the case where meta-unification coincides with unification modulo $\sim$, let us note that Drosten describes in [5] a particular meta-unification algorithm for the special case where the domains of meta-variables are $T\left(F_{0}, V\right)$ with $F_{0}$ subset of $F$.

Meta-unifiability of two meta-terms implies unifiability modulo $\sim$ of their instantiations, as a consequence of the following lemma:

Lemma 3 : Let $T$ and $T^{\prime}$ be two meta-terms and $\sigma^{\sim}$ a meta-unifier of $T$ and $T^{\prime}$. For any principal instantiation $\psi^{\prime}$ of $\sigma^{\sim}(T)$ and $\sigma^{\sim}\left(T^{\prime}\right)$, there exists a principal instantiation $\psi$ of $T$ and $T^{\prime}$ and a substitution $\sigma$ such that $\sigma . \psi \sim \psi^{\prime} \cdot \sigma^{\sim}\left[M V(T) \cup M V\left(T^{\prime}\right)\right]$. 
Note also that if $t$ and $t$ ' are terms without any meta-variable and if $\sigma^{\sim}$ belongs to Meta-Unif $\left(t, t^{\prime}\right)$, then $\phi . \sigma^{-}$is a unifier modulo $\sim$ of $t$ and $t$ ', for any instantiation $\phi$ of all the meta-variables of $\sigma^{\sim}(t)$ and $\sigma^{\sim}\left(t^{\prime}\right)$.

Now we want to unify meta-terms in the theory described by MR.

Definition 15: A substitution $\sigma^{-}$of $T(F, V U M V$ ) is a MR-meta-unifier of $T$ and $T$, (or a MR-meta-solution of the equation $\left.T==T^{\prime}\right)$ if

$-\sigma^{\sim}(T)\left\langle<=* \Rightarrow \gg \sigma^{\sim}\left(T^{\prime}\right)\right.$

- for any meta-variable $X$ in $D\left(\sigma^{\sim}\right)$ such that $d(X)=D O M_{i}$, for any instantiation $\phi$, $\phi \cdot \sigma^{\sim}(X)$ belongs to $\mathrm{DOM}_{i}$.

Again if $t$ and $t^{\prime}$ are terms without meta-variables and if $\sigma^{\sim}$ is a MR-metaunifier, for any instantiation $\phi$ of all the meta-variables of $\sigma^{\sim}(t)$ and $\sigma^{\sim}\left(t^{\prime}\right), \phi . \sigma^{\sim}$ is an Roo-unifier of $t$ and $t$ '. That results from:

Lemma 4 : If $\sigma^{\sim}$ is a MR-meta-unifier of $T$ and $T^{\prime}$, for any principal instantiation $\psi$, $\psi \cdot \sigma^{\sim}(T)<-* \rightarrow R_{\infty} \psi \cdot \sigma^{\prime}\left(T^{\prime}\right)$.

The meta-narrowing relation and meta-narrowing process are extensions of equational narrowing.

Definition 16: The meta-narrowing relation with a set of meta-rules MR, denoted $=\hat{\prime}=>$, is defined on $T(F$, UMV $)$ by: $T=^{\wedge}=>\left[u, \sigma^{\sim}, G->D\right] T$,

iff - $G->D$ belongs to $M R$

$-\sigma^{\sim}$ belongs to Meta-Unif $\left(G, T \mid u^{2}\right.$
$-T^{\prime}=T\left[u<-\sigma^{2}(D)\right]$.

As usual, to solve an equation ( $\left.t=t^{\prime}\right)$, where $t$ and $t^{\prime}$ are terms in $T(F, V)$, the meta-narrowing process consists of generating all the possible meta-narrowing derivations issued from this equation, until finding an equation on meta-terms for which a meta-unifier exists. From that derivation tree, a complete set of solutions of the equation ( $t=t^{\prime}$ ') can be found as proved in [16]. More formally:

Theorem 1 : Let $S$ be the set of substitutions $\sigma^{\prime \prime}$ of $T(F, V M V)$ such that there exists a meta-narrowing derivation from $\left(t==t^{\prime}\right.$ )

$$
t==t^{\prime} \Rightarrow \wedge\left[\sigma_{1}\right] T_{1}==T_{1}^{\prime} \Rightarrow \wedge \ldots=\wedge\left[\sigma_{n}\right] T_{n}==T^{\prime}
$$

with $T_{n}$ and $T^{\prime}{ }_{n}$ meta-unifiables, $a^{\sim}$ in Meta-Unif $\left(T_{n}=-T_{n}{ }_{n}\right)$, and $\sigma^{\sim}=a^{\sim} \cdot \sigma_{n} \ldots \ldots \sigma_{1}$.

Then $\psi(S)=\left\{\psi \cdot \sigma^{-}\right.$for any $\sigma^{\sim}$ in $S$ and any principal instantiation $\psi$ of metavariables introduced by $\left.\sigma^{\sim}\right\}$ is a complete set of Roo-unifiers of $t$ and $t$ '.

The meta-narrowing technique has been used successfully for solving equations in the signed trees theory and an adequate meta-unification algorithm has been proposed in [14]. A last example is aimed to illustrate the method.

Example 23: Let us consider the theory defined by the following axioms:

$$
\begin{aligned}
& (x * z)+(a * z)=(x+a) * z \\
& (x * y) * z=x *(y * z)
\end{aligned}
$$

where "a" is a constant. Directed from left to right, these equations generate by completion an infinite rewrite rule system, due to the superposition of associativity into the distributivity rule. The first generated rules are: 


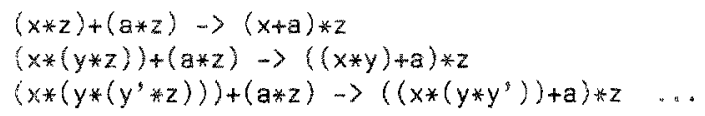

This set can be schematized by choosing for $\sim$ the equivalence modulo assaciativity, with the unconstrained meta-rule $(X * z)+(a * z) \rightarrow(X+a) * z$. Assume now that we want to solve the equation $(y+(x * a)==(a+a) * x)$ in this theory. The meta-unification coincides here with unification modulo associativity, which gives for the terms $y+(x * a)$ and $(X * z)+(a * z)$, an infinite complete set of meta-unifiers:

$$
\begin{aligned}
\Sigma= & (x \leftarrow a)(y \leftarrow x * a)(z \leftarrow a), \\
& (x \leftarrow a * a)(y \leftarrow-x *(a * a))(z \leftarrow a * a), \\
& (x \leftarrow a *(a * a))(y \leftarrow x *(a *(a * a)))(z \leftarrow a *(a * a)) \ldots 1 .
\end{aligned}
$$

A new set of meta-variables MY is then introduced, whose domain is the set of solutions of the equation $(x * a=z a * x)$ modulo associativity. The corresponding set of structures is $S=\{a, a * a, a *(a * a), a *(a *(a * a)), \ldots\}$.

The meta-narrowing reletion applied to $(y+(x * a)==(a+a) * x)$ gives then the equation $((X+a) * Y=(a+a) * Y)$ with the substitution $(x+Y)(y+X * Y)(z \leftarrow Y)$. The substitution $(X \leftarrow a)$ is a meta-unifier of the two parts of this equation. We thus get the metasolution $(y \leftarrow a * y)(x \leftarrow y)$, that generates by principal instantiations of $y$, the solutions $[(y \leftarrow a * a)(x \leftarrow a),(y \leftarrow a *(a * a))(x \leftarrow a * a),(y \leftarrow a *(a *(a * a)))(x \leftarrow a *(a * a)), \ldots\}$.

\section{Conclusion}

We propose here notions of meta-terms, meta-rewriting and meta-narrowing welladapted to the kind of problems we wanted to deal with, namely divergence of completion and completion-like processes, such as narrowing. This formalism was originally introduced in [16] and has already been widely applied in [2].

However some problems are yet unsolved, such as how to discover meta-rules. We proposed here a technique based on the generalization modulo an equivalence relation $\sim$ of an infinite sequence of terms, but this technique itself is to be studied. Another point is to find general procedures for meta-equality, meta-matching and meta-unification, problems that are handled here only in the particular cases of examples. It would be interesting to develop an approach similar to the one used in [15] for equational unification.

We have already mentioned that the notions presented here are very close to the corresponding notions used for example for handling exceptions with constrained variables as described in [5]. A main difference is that we give more general conditions for defining the domain of meta-variables, especially because we allow an equivalence relation $\sim$ on the domain of meta-variables. A second difference is that we have a notion of principal instantiation, which is not needed for defining in general metarewriting and metamarrowing, but which is very useful when we are concerned with the problem of schematization of an infinite set of rules by a finite set of meta-rules.

\section{References}

1. M.A. Ardis, "Data Abstraction Transformations," Technical Report TR-925, University of Maryland, Maryland (USA), 1980.

2. F. Bellegarde, "Utilisation des Systèmes de Réécriture d'Expressions Fonctionnelles comme outils de Transformation de Programmes Itératifs," Thèse de doctorat d'Etat, Université de Nancy I, 1985.

3. J.Y. Cras, "Conception d'un système modulaire traitant le cas de non-convergence de l'algorithme de Knuth-Bendix," Rapport de stage,Ecole Centrale des Arts et Manufactures, Chatenay-Malabry, 1983. 
4. N. Dershowitz and L. Marcus, "Existence And Construction of Rewrite Systems," Research Report, University of Illinois, USA, 1982.

5. K. Drosten, "Term Rewriting Systems with Restricted Variables," Bericht Nr.8511, Institut fur Informatik, Braunschweig, 1985.

6. M. Fay, "First-Order Unification in an Equational Theory," Proceedings of the 4th Workshop on Automated Deduction, pp. 161-167, Austin, Texas, 1979.

7. M. Hermann and I. Privara, "On nontermination of Knuth-Bendix algorithm," Research Report VUSEI-AR-OPS-3/85, Institute of Socio-Economic Information and Automation, CS-842 21 Bratislava (Czechoslovakia), 1985.

8. G. Huet, "Confluent reductions: abstract properties and applications to term rewriting systems," J. of ACM, vol. 27, no. 4, pp. 797-821, Oct. 1980.

9. G. Huet, "A complete proof of correctness of the Knuth-Bendix completion algorithm," J. Comp. Sys. Sc., vol. 23, no. 1, pp. 11-21, Aug. 1981 .

10. J.M. Hullot, "CanonicaI Forms And Unification," in Proceedings of the Fifth Conference on Automated Deduction, Lecture Notes in Computer Science, vol. 87, pp. 318-334, Springer-Verlag, Les A,rcs, France, July 1980.

11. J.Jouannaud and H.Kirchner, "Completion of a set of rules modulo a set of equations," STAM J. of Computing, vol. 15(4), 1986.

12. J.P. Jouannaud and Y. Kodratoff, "Frogram Synthesis From Example of Behaviour," Proc. of the International Workshop on Program Construction. Chateau De Bonas. Ed. Biermann And Guiho. Reiclel Publish, 1981.

13. J.P. Jouannaud, $C$. Kirchner, and $H$. Kirchner, "Incremental Construction of Unification Algorithms in Equational Theories," in Proceedings of the International Conference on Automata, Lanquages and Programming, Lecture Notes in Computer Science, vol. 154, pp. 361-3/3, Springer-Verlag, Barcelona Spain, 1983.

14. C. Kirchner and $H$. Kirchner, "Contribution à la résolution d'équations dans les algèbres libres et les variétés équationnelles d'aigèbres," Thèse de 3ème cycle, Université de Nancy 1, 1982.

15. C. Kirchner, "Méthodes et outils de conception systématique d'algorithmes d'unification dans les théories équationnelles," Thèse de doctorat d'Etat, Université de Nancy I, 1985.

16. H. Kirchner, "Preuves par complétion dans les variétés d'algèbres," Thèse de doctorat d'Etat, Université de Nancy I, 1985.

17. D. Knuth and P. Bendix, "Simple Word Problems in Universal Algebras," Computational Problems in Abstract Algebra Ed. Leech J., Pergamon Press, pp. 263-297, 1970 .

18. G. Peterson and M. Stickel, "Complete sets of reduction for equational theories with complete unification algorithms," J. of ACM, vol. 28, no. 2, pp. 233-264, 1981.

19. P. Rety, C. Kirchner, H. Kirchner, and P. Lescanne, "NARROWER: A new Algorithm for Unification and its application to Logic Programming," in Proc. Irst Conf. on Rewriting Techniques and Applications, Lecture Notes in Computer Science, vol. 202, pp. 141-157, Springer-Verlag, Dijon (France), 1985. 